Journal of Organometallic Chemistry, 172 (1979) 21-29

(C) Elsevier Sequoia S.A., Lausanne - Printed in The Netherlands

\title{
Si-C-SPALTUNG IN 2-THIENYLSILANEN DURCH SEKUNDÄRE AMINE *
}

\author{
REINHOLD TACKE * EMESE ZIMONYI-HEGEDÜS und ULRICH WANNAGAT \\ Institut für Anorganische Chemie der Technischen Universität Braunschweig, Pockelstrasse \\ 4, D-3300 Braunschweig (Bundesrepublik Deutschland)
}

(Eingegangen den 4. Januar 1979)

\section{Summary}

Treatment of diphenylvinylsilane with cyclic secondary amines (e.g. morpholine) in the presence of the corresponding lithium amides leads to substitution of the $\mathrm{SiH}$ hydrogen atom by an amino group and to addition of the amine to the vinyl group. A similar reaction observed in the case of 2-thienylphenylvinylsilane is accompanied by cleavage of the $\mathrm{Si}-\mathrm{C}$ bond and substitution of the 2-thienyl group by an amino group.

\section{Zusammenfassung}

Die Umsetzung von Diphenyl-vinylsilan mit zyklischen sekundären Aminen (z.B. Morpholin) in Gegenwart der entsprechenden Lithium-amide führt zu einer Substitution des an Silicium gebundenen H-Atoms durch eine Aminogruppe und zu einer Addition des Amins an die Vinylgruppe. 2-Thienylphenyl-vinylsilan reagiert jedoch zusätzlich unter Spaltung der Si-C-Bindung und Aminosubstitution der 2-Thienylgruppe.

\section{Einleitung}

Im Rahmen unserer Arbeitèn über Sila-Pharmaka gelang kürzlich [1] eine neue Synthese des anticholinerg wirksamen 2-Piperidinoethyldiphenylsilanols (II) gemäss Schema 1: In einer Eintopfreaktion wurde aus Diphenyl-vinylsilan mit Piperidin in Gegenwart des entsprechenden Lithiumamids zunächst 2-Piperidinoethyl-diphenyl-piperidinosilan (I) dargestellt; dieses konnte dann durch Hydrolyse in fast quantitativer Ausbeute in II übergeführt werden.

* 157. Mitt. über Beiträge zur Chemie der Sillicium-Stickstoff-Verbindungen. 156. Mitt. s. Ref. [i 4 ]. 
SCHEMA 1. Darstellung der Verbindungen $I-V$ und $I X-X I V$.
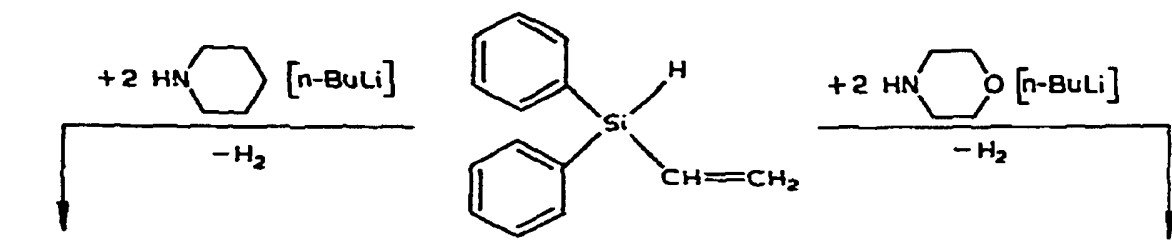

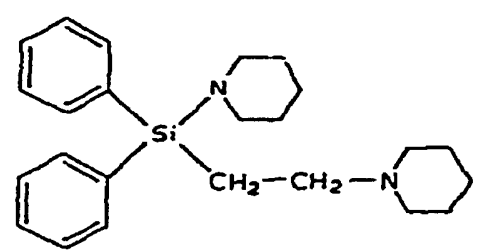

(I)

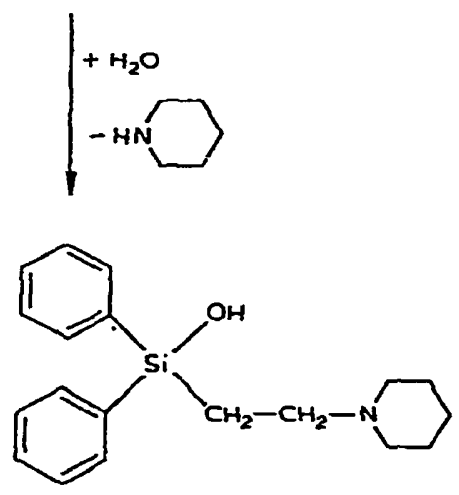

(II)

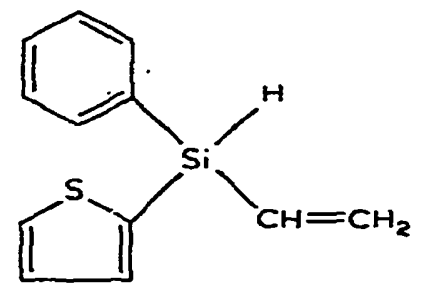

(घ्I)

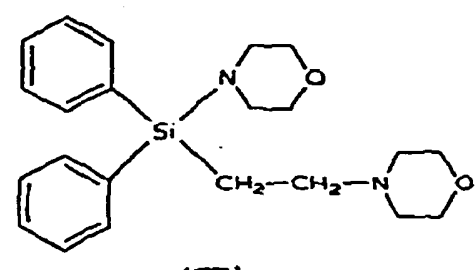

(III)

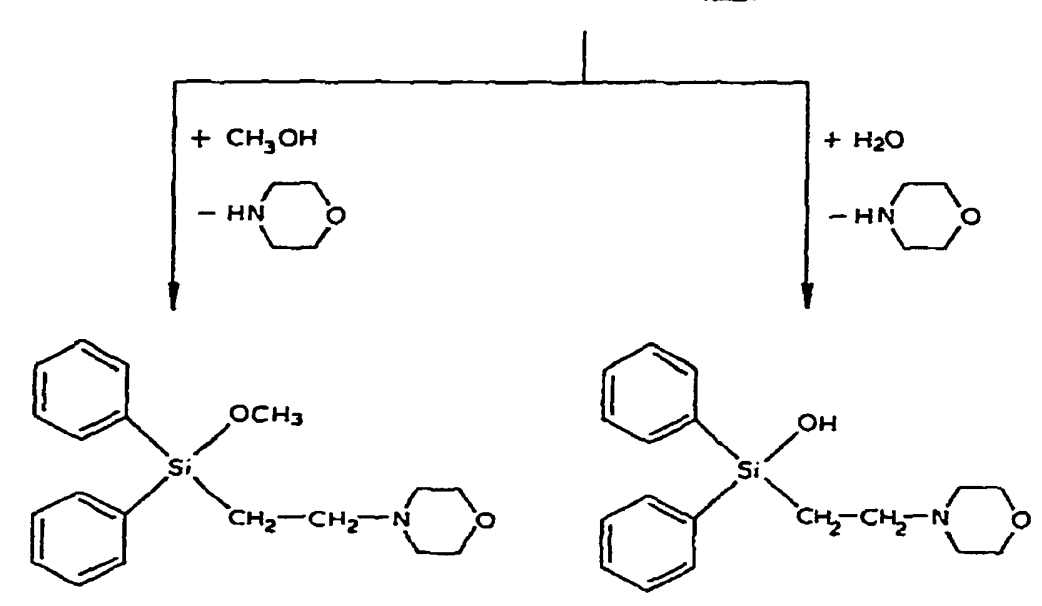

(IV)

(文)

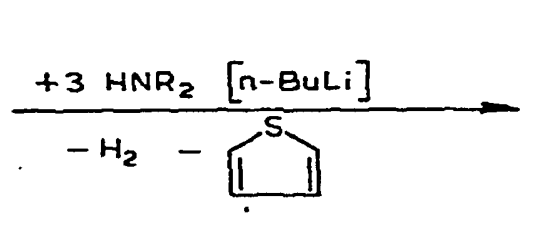

$$
\begin{aligned}
& \left(I X: N R_{2}=N O\right) \\
& \text { (XI) : } N R_{2}=N \\
& \text { (III }: N R_{2}=N \text { ) }
\end{aligned}
$$<smiles>CO[13C](O)(CC[Si](OC)(OC)OC)c1ccccc1</smiles>

$$
\begin{aligned}
& \text { ( } \mathrm{X}: \mathrm{NR}_{2}=N \\
& \text { (BII): } \mathrm{NR}_{2}=N \\
& \text { CXIV }: N R_{2}=N
\end{aligned}
$$


Eine analoge Synthese von 2-Morpholinoethyl-(2-thienyl)-phenylsilanol gelang jedoch nicht, da die Umsetzung von 2-Thienyl-phenyl-vinylsilan (VIII) mit Morpholin in Gegenwart des entsprechenden Lithiumamids unter Bruch der Si-C-Bindung zur Abspaltung der Thienylgruppe führt (vgl. Schema 1). Diese Reaktion lässt sich im Sinne einer nukleophilen Substitution der Thienylgruppe am Siliciumatom durch eine Morpholinogruppe verstehen.

In der Literatur sind bereits einige wenige Beispiele einer $\mathrm{Si}$-C-Spaltung durch Amine in Gegenwart katalytischer Mengen ihrer Amide beschrieben. Hauser und Hance [2] fanden, dass Benzyl-trimethylsilan, Diphenylmethyl-trimethylsilan und Triphenylmethyl-trimethylsilan durch Kaliumamid in flüssigem Ammoniak leicht gespalten werden. Der Si-C-Bruch erfolgt dabei zwischen dem Si-Atom und den Aralkylgruppen. Schmitz-DuMont et al. [3] berichteten, dass auch Tetraphenylsilan durch Kaliumamid in flüssigem Ammoniak gespalten wird. Benkeser et al. [4] fanden, dass Triphenylethylaminosilan durch Ethylamin in Gegenwart dessen Lithiumderivates in Diphenyl-bis(ethylamino)-silan überführt wird. Nametkin et al. [5] berichteten, dass Phenyl-dimethyl-allylsilan mit Aziridin in Gegenwart von Amid unter Entwicklung von Propen mit einer Ausbeute von etwa $10 \%$ zu Phenyl-dimethyl-aziridinosilan reagiert. Thames et al. [6] untersuchten die Reaktivität einer Reihe 2-thienyl-, phenyl-, benzyl- und allylsubstituierter Silane mit Aziridin in Gegenwart von Katalysatoren (Lithium-, Natrium- oder Kaliumsalz des Aziridins). Der 2-Furyl- wie der 2-Thienylrest erwiesen sich dabei als gute Abgangsgruppen und liessen sich in hohen Ausbeuten durch den Aziridinorest substituieren, während eine Si-C-Spaltung von Si-Phenylgruppen unter den gleichen Bedingungen nicht beobachtet werden konnte. Thames et al. [6] sehen hierin einen Hinweis für die Bedeutung einer Resonanzstabilisierung der resultierenden Carbanionen. Im Einklang hiermit steht, dass die Allylgruppensubstitution ebenfalls glatt verläuft, während sich der Benzylrest deutlich schwerer abspalten lässt als die 2-Furyl- und die 2-Thienylgruppe.

Die erhöhte Reaktivität der Si-C-Bindung in 2-Thienylsilanen wurde nicht nur in Amin/Amid-Systemen gefunden. Auch andere nukleophile Reagenzien (Hydroxidionen [7], n-Butyllithium [8]) und geeignete Elektrophile [9] vermögen die $\mathrm{Si}-\mathrm{C}-$ Bindung in 2-Thienylsilanen glatt zu spalten.

Unter diesen Gesichtspunkten wird das oben mitgeteilte unterschiedliche Reaktionsverhalten von Diphenyl-vinylsilan und 2-Thienyl-phenyl-vinylsilan gegenüber Morpholin und dessen Amid verständlich. Im folgenden wird eine Reihe von uns untersuchter $\mathrm{Si}-\mathrm{C}$-Spaltungen von 2-Thienylsilanen in Gegenwart amidhaltiger Amine beschrieben.

Ergebnisse

In Analogie zu 2-Piperidinoethyl-diphenyl-piperidinosilan (I) (vgl. Schema 1) lässt sich auch 2-Morpholinoethyl-diphenyl-morpholinosilan (III) in einer Ausbeute von etwa $85 \%$ darstellen, wobei in einer Eintopfreaktion sowohl eine Substitution des SiH-Wasserstoffs als auch eine Addition des Morpholins an die Vinylgruppe des Diphenyl-vinylsilans erfolgt. Die chemische Zusammensetzung und Struktur von III konnte durch Elementaranalysen und spektroskopische Untersuchungen ( ${ }^{1} \mathrm{H}-\mathrm{NMR}$ und MS) sowie durch chemische Umwandlung in die Verbindungen IV und V sichergestellt werden. Die Methanolyse von III führt in guten Ausbeuten durch eine $\mathrm{Si}-\mathrm{N}$-Spaltung zu dem entsprechenden 
Methoxysilan IV, während die Hydrolyse von III in fast quantitativer Ausbeute das Silanol $\mathrm{V}$ ergibt.

Im Gegensatz zu Diphenyl-vinylsilan reagiert 2-Thienyl-phenyl-vinylsilan (VIII; dessen Synthese ist in Schema 2 aufgeführt) mit Morpholin in Gegenwart des entsprechenden Lithiumderivates bei gleichen Reaktionsbedingungen unter Si-C-Spaltung (vgl. Schema 1). Es kommt nicht nur zu einer Substitution des $\mathrm{SiH}-$ Wasserstoffatoms und zu einer Addition an die Vinylgruppe, sondern ausserdem zu einer Substitution der Thienylgruppe. Das hieraus mit einer Ausbeute von $86 \%$ resultierende 2-Morpholinoethyl-phenyl-dimorpholinosilan IX konnte sowohl durch elementaranalytische und spektroskopische Untersuchungen ( $\left.{ }^{1} \mathrm{H}-\mathrm{NMR}, \mathrm{MS}\right)$ als auch durch die Überführung in das Methanolyseprodukt $\mathrm{X}$ in der chemischen Zusammensetzung und Struktur festgelegt werden.

SCHEMA 2. Darstellung dex Yerbindungen VI-VIII.<smiles>CS(Cl)(Br)c1ccc([Si](Cl)(Cl)Cl)cc1</smiles><smiles>[CH][Si](Cl)(c1ccccc1)c1cccs1</smiles>

(VI)<smiles>C=C[Si](c1ccccc1)(c1ccccc1)c1cccs1</smiles>

(III)<smiles>C=C[Si](Cl)(Cl)c1ccccc1</smiles><smiles>C=C[Si](Cl)(c1ccccc1)c1cccs1</smiles> 
Die physikalischen und chemischen Eigenschaften sowie die spektroskopischen Daten von IX und X stimmen gut mit denen authentischer Proben überein, die wir bereits durch eine andere Reaktionsfolge erhalten haben [10].

Die oben beschriebene Si-C-Spaltung ist nicht nur auf Morpholin beschränkt. Die Umsetzung von VIII mit Piperidin und Pyrrolidin in Gegenwart der entsprechenden Lithiumamide führt, ebenfalls unter $\mathrm{Si}-\mathrm{C}$-Spaltung, zu den Verbindungen XI und XIII. Sie konnten zwar nicht in analysenreiner Form isoliert, jedoch durch Methanolyse in die bereits auf anderem Wege [1] synthetisierten Methoxysilane XII und XIV übergeführt werden. Die physikalischen und chemischen Eigenschaften sowie die spektroskopischen Daten dieser beiden Verbindungen stimmen gut mit denen authentischer Proben überein.

\section{Experimentelles}

Schmelzpunkte (unkorrigiert): Kofler-Heiztischmikroskop (Fa. Reichert, Wien). Brechungsindices: Abbé-Refraktometer (Fa. Zeiss). ${ }^{1} \mathrm{H}-\mathrm{NMR}$-Spektren (90 MHz): Bruker HFX-90, Lösungsmittel $\mathrm{CDCl}_{3}$, Konzentration ca. 100 $\mathrm{mg} / \mathrm{ml}$, interner Standard TMS; die Angaben der Chemischen Verschiebungen für Multipletts beziehen sich, mit Ausnahme der aromatischen Protonen, auf die jeweiligen Zentren der $\mathrm{AA}^{\prime}$ - und $\mathrm{XX}^{\prime}$-Teile von $\mathrm{AA}^{\prime} \mathrm{XX}^{\prime}$-Systemen. Massenspektren: MS 9 (AEI, Manchester), Elektronenenergie $70 \mathrm{eV}$.

Alle Reaktionen wurden, wenn nicht anders angegeben, unter einer Schutzgasatmosphäre von trockenem Stickstoff und in absolutierten Lösungsmitteln durchgeführt.

Die Darstellung von Phenyl-dichlorsilan [11], Phenyl-vinyl-dichlorsilan [12] und Diphenyl-vinylsilan [13] erfolgte in Anlehnung an bekannte Vorschriften der Literatur.

2-Morpholinoethyl-diphenyl-morpholinosilan (III). Zu einer Lösung von $15.68 \mathrm{~g}(0.18 \mathrm{~mol})$ Morpholin in $40 \mathrm{ml}$ THF tropft man unter Rühren $18.3 \mathrm{ml}$ ( $\triangleq 0.03 \mathrm{~mol}$ BuLi) einer 15 proz. Lösung von $\mathrm{n}$-Butyllithium in $\mathrm{n}$-Hexan, rührt $30 \mathrm{~min}$ bei $40^{\circ} \mathrm{C}$ und tropft dann bei dieser Temperatur eine Lösung von 10.52 $\mathrm{g}(0.05 \mathrm{~mol})$ Diphenyl-vinylsilan in $30 \mathrm{ml}$ THF hinzu. Danach rührt man $3 \mathrm{~h}$ bei $40^{\circ} \mathrm{C}$ und $18 \mathrm{~h}$ bei $20^{\circ} \mathrm{C}$ weiter, tropft zunächst eine Lösung von $20.24 \mathrm{~g}(0.2$ mol) Triethylamin in $20 \mathrm{ml}$ Ether und dann eine Lösung von $8.69 \mathrm{~g}(0.08 \mathrm{~mol})$ Trimethylchlorsilan in $20 \mathrm{ml}$ Ether hinzu und rührt $3 \mathrm{~h}$ bei ca. $20^{\circ} \mathrm{C}$. Anschliessend filtriert man vom Niederschlag, zieht das Lösungsmittel ab und extrahiert den festen Rückstand zweimal mit je $250 \mathrm{ml}$ siedendem Petrolether $\left(40-65^{\circ} \mathrm{C}\right)$. Beim Abkühlen kristallisiert III in farblosen Kristallen aus. Ausb. $16.3 \mathrm{~g} \mathrm{(85.2 \% ).}$ Schmp. 105-107 ${ }^{\circ} \mathrm{C}$ (aus Petrolether). Elementaranalysen: Gef.: C, 68.9; H, $7.9 ; \mathrm{N}, 7.2 ; \mathrm{Si}, 7.5 . \mathrm{C}_{22} \mathrm{H}_{30} \mathrm{~N}_{2} \mathrm{O}_{2} \mathrm{Si}(382.58)$ ber.: $\mathrm{C}, 69.07 ; \mathrm{H}, 7.90 ; \mathrm{N}, 7.32$; $\mathrm{Si}, 7.34 \%$. Molmasse (massenspektrometrisch) $382 .{ }^{1} \mathrm{H}-\mathrm{NMR}(\delta, \mathrm{ppm}): 1.40$ $\left(\mathrm{m}, 2 \mathrm{H}, \mathrm{SiCH}_{2} \mathrm{C}\right), 2.43\left(\mathrm{~m}, 4 \mathrm{H}, \mathrm{CNCH}_{2} \mathrm{C}\right), 2.52\left(\mathrm{~m}, 2 \mathrm{H}, \mathrm{CCH}_{2} \mathrm{~N}\right), 2.97(\mathrm{~m}, 4 \mathrm{H}$, $\left.\mathrm{SiNCH}_{2} \mathrm{C}\right), 3.60$ und $3.69\left(\mathrm{~m}, 4 \mathrm{H}+4 \mathrm{H}, \mathrm{CCH}_{2} \mathrm{O}\right), 7.3-7.7(\mathrm{~m}, 10 \mathrm{H}$, aromatische Protonen).

Methanolyse von III; Darstellung von 2-Morpholinoethyl-diphenyl-methoxysilan (IV). $6.8 \mathrm{~g}(17.8 \mathrm{mmol})$ III werden in $25 \mathrm{ml}$ Methanol gelöst. Dann erhitzt man $20 \mathrm{~h}$ unter Rückfluss, zieht die leichtflüchtigen Anteile ab und destilliert den Rückstand fraktionierend i. Vak. über eine Vigreux-Kolonne. Ausb. $4.4 \mathrm{~g}$ 
(75.6\%), Sdp. $158-159^{\circ} \mathrm{C} / 0.4$ Torr, $n_{\mathrm{D}}^{20}$ 1.5595. Elementaranalysen: Gef.: C, $69.8 ; \mathrm{H}, 7.7 ; \mathrm{N}, 4.2 ; \mathrm{Si}, 8.7 . \mathrm{C}_{19} \mathrm{H}_{25} \mathrm{NO}_{2} \mathrm{Si}(327.50)$ ber.: $\mathrm{C}, 69.68 ; \mathrm{H}, 7.69$; $\mathrm{N}, 4.28 ; \mathrm{Si}, 8.58 \%$. Molmasse (massenspektrometrisch) $327 .{ }^{1} \mathrm{H}-\mathrm{NMR}(\delta, \mathrm{ppm})$ : $1.40\left(\mathrm{~m}, 2 \mathrm{H}, \mathrm{SiCH}_{2} \mathrm{C}\right), 2.41\left(\mathrm{~m}, 4 \mathrm{H}, \mathrm{CNCH}_{2} \mathrm{C}\right), 2.51\left(\mathrm{~m}, 2 \mathrm{H}, \mathrm{CCH}_{2} \mathrm{~N}\right), 3.54$ (s, $\left.3 \mathrm{H}, \mathrm{OCH}_{3}\right), 3.67\left(\mathrm{~m}, 4 \mathrm{H}, \mathrm{CCH}_{2} \mathrm{O}\right), 7.3-7.7(\mathrm{~m}, 10 \mathrm{H}$, aromatische Protonen)

Hydrolyse von III; Darstellung von 2-Morpholinoethyl-diphenylsilanol (V). $\mathrm{Zu}$ einer Lösung von $3.82 \mathrm{~g}(0.01 \mathrm{~mol}) \mathrm{III}$ in $100 \mathrm{ml}$ Ether gibt man $5 \mathrm{ml}$ Wasser und rührt $24 \mathrm{~h}$ bei ca. $20^{\circ} \mathrm{C}$. Dann engt man im Vakuum bei Raumtemperatur etwa auf die Hälfte des Volumens ein, lässt einige $h$ auf $-30^{\circ} \mathrm{C}$ abkühlen und isoliert schliesslich das Hydrolyseprodukt V durch Filtration. Ausb. $3.1 \mathrm{~g}$ (99\%). Umkristallisation aus Ethanol/Ether und mehrstündiges Trocknen bei $10^{-2}$ Torr liefert farblose Kristalle, die bei $160-162^{\circ} \mathrm{C}$ schmelzen. Elementaranalysen: Gef.: C, 68.9; $\mathrm{H}, 7.5 ; \mathrm{N}, 4.4 ; \mathrm{Si}, 9.0 . \mathrm{C}_{18} \mathrm{H}_{23} \mathrm{NO}_{2} \mathrm{Si}(313.48)$ ber.: $\mathrm{C}, 68.97 ; \mathrm{H}, 7.40 ; \mathrm{N}, 4.47 ; \mathrm{Si}, 8.96 \%$. Molmasse (massenspektrometrisch) 313. 'H-NMR ( $\delta, \mathrm{ppm}) ; 1.32$ ("t"t", $\left.2 \mathrm{H}, \mathrm{SiCH}_{2} \mathrm{C}\right), 2.47\left(\mathrm{~m}, 4 \mathrm{H}, \mathrm{NCH}_{2} \mathrm{C}\right), 2.67$ ("t", $2 \mathrm{H}, \mathrm{CCH}_{2} \mathrm{~N}$ ), $3.71\left(\mathrm{~m}, 4 \mathrm{H}, \mathrm{CCH}_{2} \mathrm{O}\right), \sim 5.1$ (breites Signal, nicht ganz lagekonstant, $\mathrm{H} / \mathrm{D}-\mathrm{T}$ ausch mit $\left.\mathrm{CD}_{3} \mathrm{OD}, 1 \mathrm{H}, \mathrm{SiOH}\right), 7.3-7.7(\mathrm{~m}, 10 \mathrm{H}$, aromatische Protonen).

2-Thienyl-phenyl-chlorsilan (VI). Zu $6.56 \mathrm{~g}(0.27 \mathrm{~mol})$ Magnesiumspänen und $140 \mathrm{ml}$ Ether tropft man innerhalb von 45 min eine Lösung von $42.39 \mathrm{~g}$ $(0.26 \mathrm{~mol}) 2$-Bromthiophen in $40 \mathrm{ml}$ Ether, rührt $3 \mathrm{~h}$ bei Raumtemperatur, erhitzt $1 \mathrm{~h}$ zum Rückfluss, lässt abkühlen und tropft dann die Grignardlösung bei ca. $20^{\circ} \mathrm{C}$ langsam und unter starkem Rühren zu einer Lösung von $53.1 \mathrm{~g}$ (0.3 mol) Phenyl-dichlorsilan in $500 \mathrm{ml}$ Ether. Anschliessend erhitzt man $3 \mathrm{~h}$ zum Rückfluss, rührt $24 \mathrm{~h}$ bei Raumtemperatur weiter, filtriert von den ausgefallenen Magnesiumsalzen ab, wäscht den Niederschlag dreimal mit je $50 \mathrm{ml}$ Petrolether, vereinigt das Filtrat mit den Waschlösungen, destilliert das Lösungsmittel ab und nimmt den Rückstand in $200 \mathrm{ml}$ Petrolether auf. Man lässt mehrere Stunden bei Raumtemperatur stehen, filtriert dann über eine Schicht von wasserfreiem $\mathrm{Na}_{2} \mathrm{SO}_{4}$, zieht das Lösungsmittel ab und destilliert den Rǚckstand fraktionierend im Vakuum über eine Vigreux-Kolonne. Bei $87-91^{\circ} \mathrm{C} /$ 0.1 Torr erhält man $41.5 \mathrm{~g}$ (71\% Ausbeute, bezogen auf eingesetztes 2-Bromthiopehn) von nur wenig verunreinigtem VI. Durch eine weitere Vakuumdestillation über eine Drehbandkolonne erhält man die Verbindung analysenrein. Sdp. $70^{\circ} \mathrm{C} / 0.1$ Torr, $n_{\mathrm{D}}^{20} 1.5945$. Elementaranalysen: Gef.: $\mathrm{C}, 53.8 ; \mathrm{H}, 4.1 ; \mathrm{Cl}$, 15.6; $\mathrm{S}, 14.4 ; \mathrm{Si}, 12.3$. $\mathrm{C}_{10} \mathrm{H}_{9} \mathrm{ClSSi}(224.79)$ ber.: $\mathrm{C}, 53.43 ; \mathrm{H}, 4.04 ; \mathrm{Cl}, 15.77$; $\mathrm{S}, 14.26 ; \mathrm{Si}, 12.49 \%$. Molmasse (massenspektrometrisch) 224 (bezogen auf $\left.{ }^{35} \mathrm{Cl}\right) .{ }^{1} \mathrm{H}-\mathrm{NMR}(\delta, \mathrm{ppm}): 5.84(\mathrm{~s}, 1 \mathrm{H}, \mathrm{SiH}), 7.1-7.8(\mathrm{~m}, 8 \mathrm{H}$, aromatische Protonen).

2-Thienyl-phenyl-vinyl-chlorsilan (VII). Zu einer Lösung von $121.9 \mathrm{~g}(0.6$ mol) Phenyl-vinyl-dichlorylsilan in 11 Ether tropft man bei $0^{\circ} \mathrm{C}$ innerhalb von $4 \mathrm{~h}$ unter starkem Rühren eine aus $14.6 \mathrm{~g}(0.6 \mathrm{~mol})$ Magnesiumspänen und $97.8 \mathrm{~g}(0.6 \mathrm{~mol}) 2$-Bromthiophen in $400 \mathrm{ml}$ Ether bereitete Grignardlösung. Anschliessend rührt man $12 \mathrm{~h}$ bei $20^{\circ} \mathrm{C}$ und $6 \mathrm{~h}$ unter. Rückfluss weiter, filtriert von den ausgefallenen Magnesiumsalzen, zieht das Lösungsmittel im Vakuum ab, nimmt den Rückstand in $600 \mathrm{ml}$ Petrolether auf und filtriert über eine Schicht von wasserfreiem $\mathrm{Na}_{2} \mathrm{SO}_{4}$. Dann wird der Niederschlag zweimal mit je $150 \mathrm{ml}$ Petrolether gewaschen. Man vereinigt Filtrat und Wasch- 
lösungen, dampft das Lösungsmittel im Vakuum ab und destilliert den Rückstand fraktionierend im Vakuum über eine Vigreux-Kolonne. Die Hauptfraktion, die neben VII etwas 2-Thienyl-phenyl-vinyl-bromsilan (durch Halogenaustausch entstanden) enthält, wird zwecks weiterer Reinigung einer Drehband-Destillation unterworfen. Ausbeute $70.7 \mathrm{~g}(47 \%)$ einer farblosen, hydrolyseempfindlichen Flüssigkeit. Sdp. $110^{\circ} \mathrm{C} / 0.8 \mathrm{Torr}, n_{D}^{20} 1.5940$. Elementaranalysen: Gef.: C, 57.2; $\mathrm{H}, 4.4 ; \mathrm{Cl}, 14.0 ; \mathrm{S}, 12.7 ; \mathrm{Si}, 11.4 . \mathrm{C}_{12} \mathrm{H}_{11} \mathrm{ClSSi}$ (250.82) ber.: C, 57.46; $\mathrm{H}, 4.42 ; \mathrm{Cl}, 14.13 ; \mathrm{S}, 12.78 ; \mathrm{Si}, 11.20 \%$. Molmasse (massenspektrometrisch) 250 (bezogen auf ${ }^{35} \mathrm{Cl}$ ). ${ }^{1} \mathrm{H}-\mathrm{NMR}(\delta, \mathrm{ppm}): 5.6-6.8$ $\left(\mathrm{m}, 3 \mathrm{H}, \mathrm{SiCH}=\mathrm{CH}_{2}\right), 7.1-7.7(\mathrm{~m}, 8 \mathrm{H}$, aromatische Protonen).

2-Thienyl-phenyl-vinylsilan (VIII); Umsetzung von VI mit Vinylmagnesiumchlorid. Zu einer aus $2.19 \mathrm{~g}$ (0.09 mol) Magnesiumspänen und $5.62 \mathrm{~g}(0.09 \mathrm{~mol})$ Vinylchlorid in $20 \mathrm{~g}$ THF bereiteten Grignardlösung tropft man bei ca. $20^{\circ} \mathrm{C}$ innerhalb von $45 \mathrm{~min}$ unter starkem Rühren eine Lösung von $20.23 \mathrm{~g}(0.09$ $\mathrm{mol}) \mathrm{VI}$ in $50 \mathrm{ml}$ Petrolether. Anschliessend erhitzt man $3 \mathrm{~h}$ zum Rückfluss, rührt $15 \mathrm{~h}$ bei Raumtemperatur, zieht das Lösungsmittel im Vakuum ab und versetzt den Rückstand mit Ether und gesättigter wässeriger $\mathrm{NH}_{4} \mathrm{Cl}$-Lösung. Dann trennt man die organische Phase ab, extrahiert die wässerige Phase zweimal mit je $50 \mathrm{ml}$ Ether, trocknet die vereinigten etherischen Extrakte über $\mathrm{Na}_{2} \mathrm{SO}_{4}$ und befreit vom Lösungsmittel. Eine sich anschliessende fraktionierende Vakliumdestillation über eine Vigreux-Kolonne liefert $14.3 \mathrm{~g} \mathrm{(73.4 \% )}$ von nur wenig verunreinigtem VIII. Eine weitere fraktionierende Destillation über eine Drehbandkolonne führt zu einer farblosen, analysenreinen Flüssigkeit. $\mathrm{Sdp} .91^{\circ} \mathrm{C} /$ 0.05 Torr, $n_{\mathrm{D}}^{20}$ 1.5895. Elementaranalysen: Gef.: $\mathrm{C}, 67.1 ; \mathrm{H}, 5.6 ; \mathrm{S}, 14.8 ; \mathrm{Si}$, 12.6. $\mathrm{C}_{12} \mathrm{H}_{12} \mathrm{SSi}(216.38)$ ber.: $\mathrm{C}, 66.61 ; \mathrm{H}, 5.59 ; \mathrm{S}, 14.82 ; \mathrm{Si}, 12.98 \%$. Molmasse (massenspektrometrisch) 216. ${ }^{1} \mathrm{H}-\mathrm{NMR}(\delta, \mathrm{ppm}): 5.24(\mathrm{~d}, 1 \mathrm{H}, J \sim 2.5 \mathrm{~Hz}$, $\mathrm{SiH}), 5.7-6.7\left(\mathrm{~m}, 3 \mathrm{H}, \mathrm{SiCH}=\mathrm{CH}_{2}\right), 7.1-7.7(\mathrm{~m}, 8 \mathrm{H}$, aromatische Protonen). 2-Thienyl-phenyl-vinylsilan (VIII) durch Umsetzung von VII mit LiAlH $_{4}$. Zu einer Suspension von $1.42 \mathrm{~g}(37.4 \mathrm{mmol})$ Lithiumaluminiumhydrid in $75 \mathrm{ml}$ Ether tropft man unter Rühren zügig eine Lösung von $37.62 \mathrm{~g}(150 \mathrm{mmol})$ VII in $50 \mathrm{ml}$ Ether. Man rührt $16 \mathrm{~h}$ unter Rückfluss weiter, kühlt auf $0^{\circ} \mathrm{C}$ ab und tropft dann vorsichtig $25 \mathrm{ml}$ Wasser hinzu. Anschliessend trennt man die etherische Phase ab, extrahiert die wässerige Phase dreimal mit je $50 \mathrm{ml}$ Ether, wäscht die vereinigten etherischen Extrakte mit wenig Wasser neutral, trocknet über $\mathrm{Na}_{2} \mathrm{SO}_{4}$, zieht das Lösungsmittel ab und destilliert den Rückstand im Vakuum fraktionierend über eine Vigreux-Kolonne. Ausbeute $22.6 \mathrm{~g}$ (69.8\%). Für analytische Zwecke wurde die Substanz einer zweiten fraktionierenden Vakuumdestillation unterworfen. Die physikalischen und chemischen Eigenschaften sowie die spektroskopischen Daten der Verbindung waren identisch mit denen des Produktes, das durch Umsetzung von VI mit Vinylmagnesiumchlorid erhalten wurde.

Umsetzung von VIII mit Morpholin und dessen Lithiumamid; Darstellung von 2-Morpholinoethyl-phenyl-dimorpholinosilan (IX). Zu einer Lösung von 15.68 $\mathrm{g}(0.18 \mathrm{~mol})$ Morpholin in $40 \mathrm{ml}$ THF tropft man unter Rühren $18.3 \mathrm{ml}(0.03$ mol BuLi) einer 15 proz. Lösung von n-Butyllithium in n-Hexan, rührt $30 \mathrm{~min}$ bei $40^{\circ} \mathrm{C}$, tropft dann bei dieser Temperatur eine Lösung von $10.82 \mathrm{~g}(0.05$ mol) VIII in $30 \mathrm{ml}$ THF hinzu und rührt danach $3 \mathrm{~h}$ bei $40^{\circ} \mathrm{C}$ und $18 \mathrm{~h}$ bei $20^{\circ} \mathrm{C}$ weiter. Anschliessend tropft man zunächst eine Lösung von $20.24 \mathrm{~g}$ 
(0.2 mol) Triethylamin in $20 \mathrm{ml}$ Ether und dann eine Lösung von $8.69 \mathrm{~g}(0.08$ mol) Trimethylchlorsilan in $20 \mathrm{ml}$ Ether hinzu. Man rührt $3 \mathrm{~h}$ bei $20^{\circ} \mathrm{C}$, filtriert vom Niederschlag, zieht das lösungsmittel ab und extrahiert den festen Rückstand zweimal mit je $250 \mathrm{ml}$ heissem Petrolether. Aus den vereinigten Lösungen kristallisieren nach Einengen $16.8 \mathrm{~g}(85.8 \%)$ IX, Sdp. 205-210 ${ }^{\circ} \mathrm{C} / 0.05$ Torr, Schmp. 68.5-69 $\mathrm{C}$ (Petrolether).

Methanolyse von IX; Darstellung von 2-Morpholinoethyl-phenyl-dimethoxysilan $(X)$. Man erhitzt eine Lösung von $19.58 \mathrm{~g}(0.05 \mathrm{~mol}) \mathrm{IX}$ in $100 \mathrm{ml}$ Methanol unter Rückfluss, lässt dann abkühlen, verdampft die leicht flüchtigen Anteile und destilliert den Rückstand im Vakuum fraktionierend über eine VigreuxKòlonne. Ausb. $12.8 \mathrm{~g}(91 \%)$, Sdp. $124-125^{\circ} \mathrm{C} / 0.4 \mathrm{Torr}, n_{\mathrm{D}}^{20} 1.5073$.

Umsetzung von VIII mit Piperidin und dessen Lithiumsalz zu 2-Piperidinoethyl-phenyl-dipiperidinosilan (XI) und nachfolgende Methanolyse zu 2-Piperidinoethyl-phenyl-dimethoxysilan (XII). Zu einer Lösung von $15.33 \mathrm{~g}(0.18 \mathrm{~mol})$ Piperidin in $40 \mathrm{ml}$ THF tropft man unter Rühren $18.3 \mathrm{ml}(0.03 \mathrm{~mol} \mathrm{BuLi})$ einer 15proz. Lösung von n-Butyllithium in n-Hexan, rührt $30 \mathrm{~min}$ bei $40^{\circ} \mathrm{C}$, tropft dann bei dieser Temperatur eine Lösung von $10.82 \mathrm{~g}(0.05 \mathrm{~mol})$ VIII in $30 \mathrm{ml}$ THF hinzu und rührt $3 \mathrm{~h}$ bei $40^{\circ} \mathrm{C}$ und $18 \mathrm{~h}$ bei $20^{\circ} \mathrm{C}$ weiter. Anschliessend tropft man zunächst eine Lösung von $20.24 \mathrm{~g}(0.2 \mathrm{~mol})$ Triethylamin in $20 \mathrm{ml}$ Ether und dann eine Lösung von $8.69 \mathrm{~g}$ (0.08 mol) Trimethylchlorsilan in $20 \mathrm{ml}$ Ether hinzu. Man rührt $3 \mathrm{~h}$ bei $20^{\circ} \mathrm{C}$, filtriert vom Niederschlag, zieht das Lösungsmittel ab und destilliert den Rückstand im Vakuum über eine Vigreux-Kolonne.

Bei $170-185^{\circ} \mathrm{C} / 0.2$ Torr erhält man $17.4 \mathrm{~g}$ einer hauptsächlich aus XI bestehenden Fraktion. Diese löst man in $80 \mathrm{ml}$ Methanol und erhitzt $5 \mathrm{~h}$ zum Rückfluss. Dann engt man ein und unterwirft den Rückstand einer fraktioniererden Vakuumdestillation. Ausb. $9.6 \mathrm{~g}$ XII (68.7\%, bezogen auf eingesetztes VIII), Sdp. $95^{\circ} \mathrm{C} / 0.02$ Torr, $n_{\mathrm{D}}^{20} 1.5058$.

Die Umsetzung von VIII mit Pyrrolidin und dessen Lithiumamid zu 2-Pyrrolidinoethyl-phenyI-dipyrrolidinosilan (XIII) sowie nachfolgende Methanolyse zu 2-Pyrroilidinoethyl-phenyl-dimethoxysilan (XIV). Diese Reaktion erfolgte in Analogie zur Darstellung von XI und XII. Man erhält bei $140-155^{\circ} \mathrm{C} / 0.2$ Torr 14.9 g einer Fraktion, die grösstenteils aus XIII besteht. Die anschliessende Methanolyse liefert $9.3 \mathrm{~g}$ XIV (70\% Ausbeute, bezogen auf eingesetztes VIII), Sdp. $91-92^{\circ} \mathrm{C} / 0.01$ Torr, $n_{D}^{20} 1.5045$.

Dank

Wir danken dem Verband der Chemischen Industrie, Frankfurt am Main, sowie dem Land Niedersachsen für die Förderung durch Forschungsmittel, der Bayer AG, Leverkusen, für die Überlassung von Chlorsilanen.

\section{Literatur}

1 R. Tacke und M. Strecker, in Vorbereitung.

2 C.R. Hauser und C.R. Hance, J. Amer. Chem. Soc., 73 (1951) 5846.

3 O. Schmitz-DuMont, W. Jansen und W. Schaal, Z. Anorg. Allg. Chem., 339 (1965) 113.

4 R.A. Benkeser, R.E. Robinson und H. Landesman, J. Amer. Chem. Soc., 74 (1952) 5699.

5 N.S. Nametkin, I.A. Grushevenko und V.N. Perchenko, Izv. Akad. Nauk SSSR, Ser. Khim., (1966) 737; Chem. Abstr., 65 (1966) 8947. 
6 S.F. Thames, J.E. McClesky und P.L. Kelly, J. Heterocyclic Chem., 5 (1968) 749.

7 R.H. Meen und H. Gilman, J. Org. Chem., 20 (1955) 73.

8 F.H. Pinkerton und S.F. Thames, J. Organometal. Chem., 29 (1971) C4.

9 V. Bažant, V. Chvalovský und J. Rathouský, Organosilicon Compounds, Vol. 1, Academic Press, New York, 1965, S. 232.

10 R. Tacke und E. Zimonyi-Hegedüs, in Vorbereitung.

11 H.J. Emeleus und S.R. Robinson, J. Chem. Soc., (1947) 1592.

12 S.D. Rosenberg, J.J. Walbum und H.E. Ramsden, J. Org. Chem., 22 (1957) 1606.

13 J.W. Curry, J. Amer. Chem. Soc., 78 (1956) 1686.

14 D.J. Brauer, H. Bürger, H.H. Moretto, U. Wannagat und K. Wiegel, J. Organometal. Chem., im Druck. 